\title{
Severely reduced diffusion capacity in idiopathic pulmonary arterial hypertension: patient characteristics and treatment responses
}

\author{
Pia Trip ${ }^{1}$, Esther J. Nossent ${ }^{1}$, Frances S. de Man ${ }^{1,2}$, Inge A.H. van den Berk ${ }^{3}$, \\ Anco Boonstra', Herman Groepenhoff ${ }^{1}$, Edward M. Leter ${ }^{4}$, Nico Westerhof ${ }^{1}{ }^{1}$, \\ Katrien Grünberg ${ }^{5}$, Harm-Jan Bogaard ${ }^{1}$ and Anton Vonk-Noordegraaf ${ }^{1}$
}

Affiliations: ${ }^{1}$ Dept of Pulmonary Medicine, Institute for Cardiovascular Research, VU University Medical Center, Amsterdam, ${ }^{2}$ Dept of Physiology, Institute for Cardiovascular Research, VU University Medical Center, Amsterdam, ${ }^{3}$ Dept of Radiology, Academic Medical Center, Amsterdam, ${ }^{4}$ Dept of Clinical Genetics, Institute for Cardiovascular Research, VU University Medical Center, Amsterdam, and ${ }^{5}$ Dept of Pathology, Institute for Cardiovascular Research, VU University Medical Center, Amsterdam, The Netherlands.

Correspondence: A. Vonk-Noordegraaf, VU University Medical Center, Dept of Pulmonary Medicine, De Boelelaan 1117, 1081 HV Amsterdam, The Netherlands. E-mail: a.vonkdvumc.nl

ABSTRACT A subgroup of patients with idiopathic pulmonary arterial hypertension (IPAH) has severely reduced diffusing capacity of the lung for carbon monoxide (DLCO) and poor prognosis. Their characteristics are currently unknown. The aim of this study is to contrast clinical characteristics and treatment responses of IPAH-patients with a severely reduced and more preserved DLCO.

Retrospectively, 166 IPAH patients were included and grouped based on a DLCO cut-off value of $45 \%$ pred $\left(\mathrm{IPAH}_{<45 \%}\right.$ and $\left.\mathrm{IPAH} \mathrm{H}_{\geqslant 45 \%}\right)$. Clinical characteristics, treatment responses and survival were compared.

$\mathrm{IPAH}_{<45 \%}$ were older, more often male, had a more frequent history of coronary disease and a higher tobacco exposure. Forced expiratory volume in $1 \mathrm{~s}$ (FEV1), FEV1/forced vital capacity, total lung capacity and alveolar volume values were slightly lower and computed tomography scan abnormalities more prevalent in patients with a low DLCO. Age and number of pack years were independently associated with DLCO $<45 \%$ pred. IPAH I $_{<5 \%}$ showed no different haemodynamic profile, yet worse exercise performance and a worse survival rate, which were both related to age, sex and the presence of coronary disease.

To conclude, a severely reduced DLCO in IPAH is associated with advanced age and a greater tobacco exposure. These patients have a worse exercise performance despite a similar hemodynamic profile. We confirm the decreased survival in this patient group and now show that this poor outcome is related to age, sex and the presence of coronary disease.

@ERSpublications

Severely reduced DLCO in IPAH is associated with advanced age and a greater tobacco exposure http://ow.ly/pkQ5F

Earn CME accreditation by answering questions about this article. You will find these at the back of the printed copy of this issue or online at www.erj.ersjournals.com/misc/cmeinfo.xhtml

Received: Nov 142012 | Accepted after revision: July 302013 | First published online: Aug 152013

Support statement: P. Trip and A. Vonk-Noordegraaf were supported by a grant from the Netherlands Organization for Scientific Research (NWO)-VIDI project number 917.96.306.

Conflict of interest: Disclosures can be found alongside the online version of this article at www.erj.ersjournals.com

Copyright @ERS 2013 


\section{Introduction}

In about $75 \%$ of patients with idiopathic pulmonary arterial hypertension (IPAH), the diffusing capacity of the lung for carbon monoxide (DLCO) is reduced [1]. The reduction in DLCO is moderate in the majority of patients and the presence of a severely reduced DLCO during the diagnostic work-up should raise suspicion of secondary causes of pulmonary hypertension, such as connective tissue disease $[2,3]$, pulmonary venoocclusive disease [4], left heart failure [5], and parenchymal lung disease [6, 7]. However, in a subgroup of patients no secondary causes explaining the low DLCO are found and patients are then classified as IPAH.

IPAH patients with a severely reduced DLCO have a significantly worse survival $[8,9]$. Nevertheless, the clinical characteristics of this subgroup of IPAH-patients were never described and the factors associated with a severely reduced DLCO remain unknown. A recent cohort study on IPAH patients provides some insight into the factors that may play a role in the reduction of DLCO. LiNG et al. [10] compared two different IPAH age groups and showed that older IPAH patients show a different IPAH phenotype with lower DLCO values, a more frequent history of smoking, ischaemic heart disease, hypertension, diabetes, and a worse survival rate when compared with younger patients. This raises the question whether age, smokingrelated lung disease, or cardiovascular comorbidities are factors playing a role in reducing DLCO.

The proportion of IPAH patients with advanced age, lower DLCO and a worse prognosis is increasing $[10,11]$, and a more detailed study of this subgroup is needed. In this study we aim to determine the factors that contribute to a severely reduced DLCO and contrasted the clinical characteristics of IPAH patients with a severely reduced DLCO to those of IPAH patients with a more preserved DLCO. In addition, we compared treatment responses and survival between the two groups.

\section{Methods}

Study design and patient selection

We retrospectively studied IPAH and heritable PAH (HPAH) patients consecutively seen at the VU University Medical Center (Amsterdam, the Netherlands) between January 1990 and November 2011. Patients were included when, after clinical evaluation, the multidisciplinary team agreed upon a diagnosis of IPAH or HPAH. Clinical evaluation included: echocardiography, high-resolution computed tomography (HRCT) of the chest, pulmonary function testing, and right heart catheterisation. For the purpose of this study, a re-evaluation of the chest CT scans was performed by a radiologist blinded to the initial diagnosis. The presence of emphysema and/or fibrosis was quantified using a three-point scale. Mild emphysema was defined by subtle centrilobular emphysema in apical segments of upper lobes; moderate emphysema was defined by a cluster of centrilobular and paraseptal emphysema, with a preference for upper lobes; and severe emphysema was defined by generalised centrilobular and paraseptal emphysema in both upper and lower lobes. Mild fibrosis was defined by focal areas with fine reticular subpleural opacities; moderate fibrosis was defined by a continuous subpleural band of fine reticular opacities, which were restricted to either the upper or lower lobes; and severe fibrosis was defined by subpleural reticular opacities stretching along both the upper and lower part of both lungs.

IPAH and HPAH were diagnosed in patients with a mean pulmonary arterial pressure (PAP) $\geqslant 25 \mathrm{mmHg}$ and pulmonary capillary wedge pressure (PCWP) $\leqslant 15 \mathrm{mmHg}$, not explained by an underlying parenchymal lung disease or chronic obstructive pulmonary disease (COPD) [12].

Not included in this study were patients diagnosed with pulmonary veno-occlusive disease (PVOD), figure 1 provides more details. IPAH or HPAH patients aged $<18$ years at the time of diagnosis were excluded, as were patients with a forced expiratory lung volume after $1 \mathrm{~s}$ (FEV1) or forced expiratory capacity (FVC) of $<60 \%$ pred (fig. 1). In addition, IPAH and HPAH patients with severe emphysema and/ or severe fibrosis on re-evaluation of the chest CT were excluded. The total study cohort consisted of 166 IPAH and HPAH patients (which will subsequently be referred to as IPAH, unless otherwise stated) and was divided into a group with a severely reduced DLCO $\left(\mathrm{IPAH}_{<45 \%}\right)$ and a group with a more preserved DLCO $\left(\mathrm{IPAH}_{\geqslant 45 \%}\right)$. The cut-off value of $45 \%$ pred was based on the frequency distribution of DLCO values.

\section{Clinical characteristics}

Data on demographics, smoking history, medical history, medication usage and World Health Organization functional class (WHO FC), were all taken at the time of diagnosis. Coronary artery disease was indicated as present if the patient's history mentioned myocardial infarction, percutaneous transluminal coronary angioplasty or coronary artery bypass surgery.

Routine laboratory test results taken within half a year from diagnosis were analysed. Auto-immune serology and pulmonary function test results were collected at the time of diagnosis or, when not present at baseline, the first result taken during follow-up was used (median follow-up duration 1 day, interquartile range (IQR) -5-167 days). Spirometry, bodyplethysmography, and single-breath DLCO were measured in 
a)
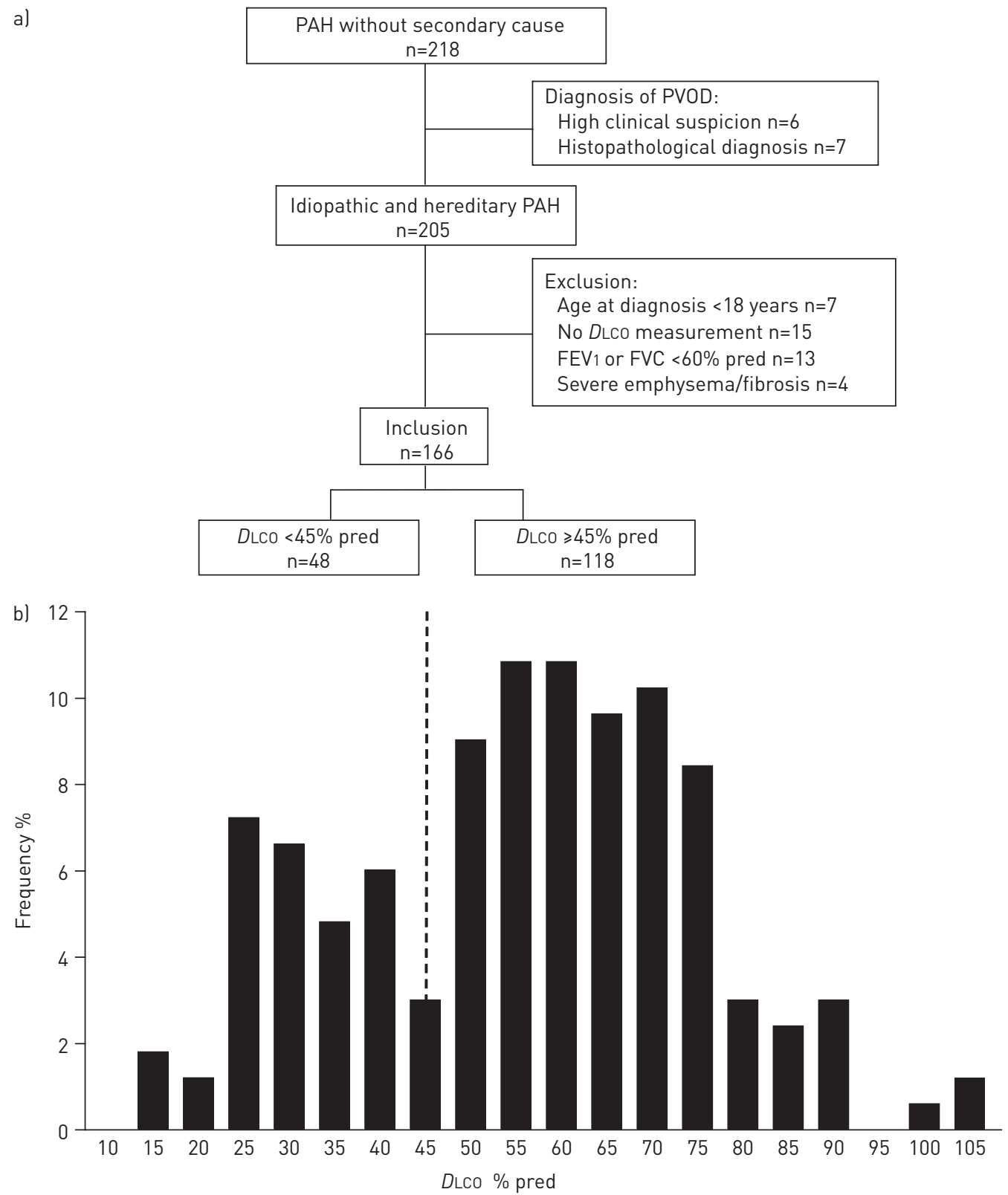

FIGURE 1 a) Flowchart displaying the inclusion for idiopathic and hereditary pulmonary arterial hypertension (I/HPAH) patients and b) their respective diffusing capacity of the lung for carbon monoxide (DLCO) frequency distribution. A total of 218 patients with $\mathrm{PAH}$, without a secondary cause, were seen in our hospital. Patients diagnosed with pulmonary venoocclusive disease (PVOD), based on either a high clinical suspicion or a pathological diagnosis of PVOD after lung biopsy or at autopsy, were excluded. A high clinical suspicion of PVOD was present if a severely reduced DLCO was accompanied by two or three of the following abnormalities using high-resolution computed tomography (CT): centrilobular groundglass opacities, septal lines, or mediastinal lymph node enlargement [13]. 13 patients were diagnosed with PVOD based on a high clinical suspicion $(n=6)$ and a histopathological diagnosis $(n=7)$. A total of 205 I/HPAH patients were enrolled in this study. Seven patients aged $<18$ years at the time of diagnosis were excluded. I/HPAH patients with a forced expiratory volume in $1 \mathrm{~s}$ (FEV1) or forced expiratory capacity (FVC) $<60 \%$ pred were also excluded; as we aimed to obtain a homogenous patient population and these patients did not meet the inclusion criteria of most randomised clinical trials on PAH-targeted therapy [14]. Three IPAH patients were excluded due to the presence of severe emphysema on a CT-scan re-evaluation, and one HPAH patient was excluded due to the presence of severe fibrosis. In 15 patients DLCO measurements were not available. The total study cohort consisted of 166 patients that were divided into a severely reduced DLCO group (IPAH $<45 \%$ ) and a more preserved DLCO group ( $\mathrm{IPAH}_{<45 \%}$ ) based on the frequency distribution of DLCO values using a cut-off of $45 \%$ pred. 
accordance with the European Respiratory Society guidelines $[15,16]$. To determine whether a change in DLCO could be observed during follow-up, subsequent pulmonary function tests were collected and compared with baseline values. 6-min walk test (6MWT) results were collected within 6 months from diagnosis.

$6 \mathrm{MWT}$ results included the 6-min walking distance $(6 \mathrm{MWD})$, the $6 \mathrm{MWD}$ as percentage of predicted, arterial oxygen saturation $\left(\mathrm{SaO}_{2}\right)$ at rest and decrease in arterial oxygen saturation during exercise.

Results from right heart catheterisation were taken at baseline. Total pulmonary vascular resistance (TPVR) was calculated as mean PAP divided by cardiac output. Arterial blood gas measures included $\mathrm{pH}$, arterial carbon dioxide tension $\left(\mathrm{PCO}_{2}\right)$, arterial oxygen tension, and $\mathrm{SaO}_{2}$.

Final diagnosis was either IPAH or HPAH. Heritable PAH included clinical familial cases with or without identified germline BMPR2 gene mutations as well as clinically sporadic IPAH-patients with an identified germline BMPR2 gene mutation [12].

\section{Treatment response and survival}

First-line treatment was given according to contemporary guidelines and consisted of an endothelin receptor antagonist, a prostanoid, a phosphodiesterase type-5 inhibitor, a calcium channel blocker, or combination treatment. To assess differences in treatment responses, time to clinical worsening (TTCW; defined as time to add-on PAH-specific therapy, atrial balloon septostomy, lung transplantation or death) was compared between the groups.

Follow-up was not conducted until May 1, 2012. Instead of overall survival we used event-free survival with lung transplantation as an additional end-point. We did this because IPAH patients who receive a lung transplant have end-stage disease and an expected survival of $<4$ months. Therefore, by using event-free survival we minimised the bias introduced by not taking into account lung transplantation. Event-free survival was calculated from the time of diagnosis with all-cause mortality or lung transplantation as an end-point.

\section{Statistical analysis}

Categorical and continuous variables were compared by binary logistic regression analysis and linear regression analysis, respectively, while correcting for age. Continuous variables that are presented as percentage of predicted, and thus corrected for age, were compared by unpaired T-tests or Mann-Whitney U-tests. First-line treatment was compared by a chi-squared test. A p-value of $<0.05$ was considered significant.

A backward stepwise multivariate logistic regression analysis was performed to determine the characteristics that were independently associated with a severely reduced DLCO. Variables included into the analysis were age, sex, body mass index, smoking, pack years, coronary disease, FEV1, FEV1/FVC, total lung capacity (TLC), and alveolar volume (VA). Survival was analysed with the Kaplan-Meier method and compared by the log-rank test. To correct the association between event-free survival and a severely reduced DLCO for age, sex, time between the DLCO measurement and diagnosis and the presence of coronary disease a cox proportional hazards regression analysis was used. The association between TTCW and a severely reduced DLCO, while correcting for age, was analysed by a cox proportional hazards regression analysis.

\section{Results}

48 patients had a $D$ LCO $<45 \%$ pred $\left(\right.$ IPAH $\left._{<45 \%}\right)$ and 118 patients had a DLCO $\geqslant 45 \%$ pred $\left(\right.$ IPAH $\left._{<45 \%}\right)$. Table 1 shows the clinical characteristics of the two DLCO groups. IPAH $\mathrm{P}_{<45 \%}$ were older, more often male and had a more frequent smoking history. The number of pack years could be acquired in 33 out of 48 $\mathrm{IPAH}_{<45 \%}$ patients, and 93 out of $118 \mathrm{IPAH}_{\geqslant 45 \%}$ patients. A higher number of pack years was observed in the $\mathrm{IPAH}_{<45 \%}$ patients. In $\mathrm{IPAH}_{<45 \%}$ an increased prevalence of coronary heart disease was found. No differences were observed in the prevalence of hypertension, thyroid disease, malignancy or pulmonary diseases, such as mild COPD, asthma and mild OSAS.

The comparison of medication usage revealed no difference in the use of anticoagulants, antihypertensive medications, statins, nitrates and bronchodilators in $\mathrm{IPAH}_{<45 \%}$ when compared to $\mathrm{IPAH}_{\geqslant 45 \%}$. The WHO FC, at the time of diagnosis, was similar in both groups. Pulmonary function testing revealed lower FEV1, FEV1/FVC, TLC and VA-values in $\mathrm{IPAH}_{<45 \%}$. CT-scans were available for re-analysis in 140 patients. Results are shown in table 2. Abnormalities were more often seen in $\mathrm{IPAH}_{<45 \%}(68 \%$ versus $33 \%$ in $\left.\mathrm{IPAH}_{\geqslant 45 \%} ; \mathrm{p}=0.004\right)$. The majority of IPAH $<45 \%$ patients with abnormalities on a CT $(57 \%)$ showed mild or moderate emphysema without any signs of fibrosis. No $\mathrm{IPAH}_{<45 \%}$ patients presented with moderate emphysema in combination with moderate fibrosis. Blood test results showed similar haemoglobin levels in both the IPAH groups, but lower $\mathrm{PCO}_{2}$ and $\mathrm{Sa}_{2} \mathrm{O}_{2}$ in $\mathrm{IPAH}_{<45 \%}$ Arterial $\mathrm{PCO}_{2}$ was low compared to reference values in both IPAH groups and was not associated with DLCO $(r=0.17)$. Results from autoimmune serology testing were available in $34 \mathrm{IPAH}_{<45 \%}(71 \%)$ and $81 \mathrm{IPAH}_{\geqslant 45 \%}$ patients $(69 \%)$. The results are 
TABLE 1 Clinical characteristics and final diagnosis according to the diffusing capacity of the lung for carbon monoxide (DLCO)

\begin{tabular}{|c|c|c|c|}
\hline Demographics & DLCO $<45 \%$ pred & DLCO $\geqslant 45 \%$ pred & p-value \\
\hline Patients n & 48 & 118 & \\
\hline Age at diagnosis years & $67(53-75)$ & $46(35-60)$ & $<0.001$ \\
\hline Sex male & $24(50)$ & $22(19)$ & 0.013 \\
\hline Body mass index $\mathrm{kg} \cdot \mathrm{m}^{-2}$ & $26 \pm 4$ & $27 \pm 6$ & 0.035 \\
\hline Smoking & $33(77)$ & $54(48)$ & 0.033 \\
\hline Current smoker & 8 (19) & $20(18)$ & 0.765 \\
\hline Former smoker & $25(58)$ & $34(30)$ & 0.065 \\
\hline Pack years & $25(0-40)$ & $0(0-13)$ & 0.009 \\
\hline \multicolumn{4}{|l|}{ Medical history } \\
\hline Coronary disease & $13(27)$ & $1(1)$ & 0.008 \\
\hline Hypertension & $14(29)$ & $26(22)$ & 0.207 \\
\hline Diabetes mellitus & $12(25)$ & $11(9)$ & 0.513 \\
\hline Thyroid disease & $4(8)$ & $12(10)$ & 0.701 \\
\hline \multicolumn{4}{|l|}{ Pulmonary disease } \\
\hline COPD (GOLD I-II) & $5(10)$ & $6(5)$ & 0.845 \\
\hline OSAS & $0(0)$ & $2(2)$ & 0.997 \\
\hline Asthma & $0(0)$ & $9(8)$ & 0.997 \\
\hline Malignancy & $6(13)$ & $10(9)$ & 0.678 \\
\hline \multicolumn{4}{|l|}{ Medication use } \\
\hline Diuretics & $20(43)$ & 38 (33) & 0.730 \\
\hline Anticoagulants & $31(66)$ & 45 (39) & 0.215 \\
\hline Antihypertensive therapy & $31(66)$ & $42(36)$ & 0.304 \\
\hline Statins & $16(34)$ & $10(9)$ & 0.075 \\
\hline Nitrates & $9(19)$ & $4(3)$ & 0.085 \\
\hline Bronchodilator therapy & $12(26)$ & $12(10)$ & 0.110 \\
\hline Corticosteroids & $10(21)$ & $20(17)$ & 0.567 \\
\hline Oxygen & $5(11)$ & $5(4)$ & 0.517 \\
\hline WHO functional class & & & $0.050^{\#}$ \\
\hline I & $0(0)$ & $6(6)$ & \\
\hline II & $9(21)$ & $32(29)$ & \\
\hline III & $27(61)$ & $61(56)$ & \\
\hline IV & $8(18)$ & $10(9)$ & \\
\hline \multicolumn{4}{|l|}{ Pulmonary function } \\
\hline FEV1 \% pred & $85 \pm 16$ & $91 \pm 16$ & $0.010^{\top}$ \\
\hline FVC \% pred & $99 \pm 16$ & $102 \pm 17$ & $0.206^{\top}$ \\
\hline $\mathrm{FEV}_{1} / \mathrm{FVC} \%$ & $68 \pm 9$ & $74 \pm 9$ & $<0.001^{\circ}$ \\
\hline TLC \% pred & $92 \pm 16$ & $99 \pm 12$ & $0.004^{\circ}$ \\
\hline VA $\%$ pred & $78 \pm 14$ & $85 \pm 11$ & $0.001^{\top}$ \\
\hline \multicolumn{4}{|l|}{ Laboratory tests } \\
\hline Haemoglobin $\mathrm{mmol} \cdot \mathrm{L}^{-1}$ & $9.5 \pm 1.1$ & $9.1 \pm 1.1$ & 0.236 \\
\hline Creatinine $\mathrm{umol} \cdot \mathrm{L}^{-1}$ & $99 \pm 29$ & $95 \pm 22$ & 0.757 \\
\hline Ureum mmol $\cdot \mathrm{L}^{-1}$ & $6.9(4.9-8.4)$ & $5.9(4.7-7.9)$ & 0.127 \\
\hline NT-proBNP $n g \cdot L^{-1}$ & $999(204-2266)$ & 732 (214-2934) & 0.765 \\
\hline \multicolumn{4}{|l|}{ Arterial blood gas } \\
\hline $\mathrm{pH}$ & $7.45 \pm 0.03$ & $7.45 \pm 0.04$ & 0.368 \\
\hline $\mathrm{PCO}_{2} \mathrm{~mm} \mathrm{Hg}$ & $31 \pm 6$ & $32 \pm 6$ & 0.015 \\
\hline $\mathrm{PO}_{2} \mathrm{~mm} \mathrm{Hg}$ & $62 \pm 16$ & $72 \pm 13$ & 0.153 \\
\hline $\mathrm{SaO}_{2} \%$ & $91 \pm 5$ & $94 \pm 3$ & 0.024 \\
\hline Final diagnosis & & & $<0.001^{\circ}$ \\
\hline Non-heritable IPAH & 48 (100) & $91(77)$ & \\
\hline Heritable PAH & $0(0)$ & 27 (23) & \\
\hline
\end{tabular}

Data are presented as median (25th-75th percentile), $\mathrm{n}(\%)$ or mean \pm SD, unless otherwise stated. COPD: chronic obstructive pulmonary disease; GOLD: Global initiative for Chronic Obstructive Lung Disease class; OSAS: obstructive sleep apnoea syndrome; WHO: World Health Organization; FEV1: forced expiratory volume in $1 \mathrm{~s}$; FVC: forced vital capacity; TLC: total lung capacity; VA, alveolar volume; NT-proBNP: N-terminal brain natriuretic peptide; $\mathrm{PCO}_{2}$ : arterial carbon dioxide tension; $\mathrm{PO}_{2}$ : arterial oxygen tension; $\mathrm{SaO}_{2}$ : arterial oxygen saturation; IPAH: idiopathic pulmonary arterial hypertension. The $\mathrm{p}$-values are from binary logistic or linear regression analysis corrected for age. "\# : p-value based on WHO functional class I-II versus III-IV. ๆ: uncorrected p-value. 
TABLE 2 Computed tomography findings in patients according to the diffusing capacity of the lung for carbon monoxide (DLCo) Presence of fibrosis

Presence of emphysema

\begin{tabular}{|c|c|c|c|c|c|c|}
\hline & \multicolumn{3}{|c|}{ DLco $<45 \%$ pred $^{\#}$} & \multicolumn{3}{|c|}{ DLCO $\geqslant 45 \%$ pred } \\
\hline & None & Mild & Moderate & None & Mild & Moderate \\
\hline Mild & $3(7)$ & $4(9)$ & $4(9)$ & $1(1)$ & $1(1)$ & $1(1)$ \\
\hline Moderate & $0(0)$ & 2 (5) & $0(0)$ & $1(1)$ & $0(0)$ & $0(0)$ \\
\hline
\end{tabular}

Data are presented as are $n(\%) .{ }^{\#}: n=44 ;{ }^{\bullet}: n=96$. Computed tomography scan analysis was performed by a blinded radiologist. Mild emphysema was defined by subtle centrilobular emphysema in apical segments of upper lobes; moderate emphysema was defined by a cluster of centrilobular and paraseptal emphysema with a preference for upper lobes; mild fibrosis was defined by focal areas with fine reticular subpleural opacities; moderate fibrosis was defined by a continuous subpleural band of fine reticular opacities restricted to either the upper or lower lobes.

shown in table 3. The presence of antinuclear antibodies (ANA) tended to be increased in $\mathrm{IPAH}_{<45 \%}$. Although genetic tests were performed in 68 of the IPAH patients, it is of interest that a final diagnosis of heritable IPAH was only made in patients with a preserved DLCO (table 1).

Table 4 shows the clinical characteristics associated with a severely reduced DLCO on multivariate regression analysis. Age and the number of pack years were independently associated with a severely reduced DLCO.

\section{Exercise performance and haemodynamics}

Figure 2 shows baseline haemodynamic results. $\mathrm{IPAH}_{<45 \%}$ had similar mean PAP when compared to $\mathrm{IPAH}_{\geqslant 45 \%}$. Also mean right atrial pressure, cardiac index (CI), TPVR, PCWP and mixed venous oxygen saturation $\left(\mathrm{SvO}_{2}\right)$ were not different between the two groups.

In $77 \%$ and $72 \%$ of $\mathrm{IPAH}_{<45 \%}$ and $\mathrm{IPAH}_{\geqslant 45 \%}$ patients, respectively, baseline $6 \mathrm{MWT}$ results were available. The results are shown in figure 3 . $\mathrm{IPAH}_{<45 \%}$ had a lower $6 \mathrm{MWD}$ when compared with $\mathrm{IPAH}_{\geqslant 45 \%}$. Moreover, $\mathrm{IPAH}_{<45 \%}$ had a greater decrease in $\mathrm{SaO}_{2}$ during the test.

\section{Treatment response and survival}

Table 5 shows first-line treatments and treatment responses. First-line treatment was started in $99 \%$ of IPAH patients. The remaining two patients died before receiving treatment. No differences in treatment choices could be observed. TTCW was similar for $\mathrm{IPAH}_{<45 \%}$ and $\mathrm{IPAH}_{\geqslant 45 \%}$ when corrected for age.

In $23 \mathrm{IPAH}_{<45 \%}$ patients and $76 \mathrm{IPAH}_{\geqslant 45 \%}$ patients a follow-up DLCO measurement was available. Time between the first and follow-up DLCO measurement was not different between $\mathrm{IPAH}_{<45 \%}$ (median 416 days, IQR 316-834 days) and IPAH $\geqslant 45 \%$ (median 447 days, IQR 368-1008 days; p=0.35). No difference could be observed between the first DLCO measurement (DLCO1) and follow-up measurement $\left(D\right.$ LCO2) in either group $\left(\mathrm{IPAH}_{<45 \%}\right.$, DLCO $33 \pm 7 \%$ pred and DLCO2 $34 \pm 8 \%$ pred, $\mathrm{p}=0.42$; and $\mathrm{IPAH}_{\geqslant 45 \%}$, DLCO1 $67 \pm 13 \%$ pred and DLCO2 $67 \pm 13 \%$ pred, p 0.95).

Time of follow-up could not be acquired in two $\mathrm{IPAH}_{<45 \%}$ patients and eight $\mathrm{IPAH}_{\geqslant 45 \%}$ patients. Median follow-up time was 4.2 years (IQR 1.8-7.9 years). During this period 53 events occurred. 10 patients had undergone lung transplantation. Cause of death was retrieved in 25 patients. 18 patients had died due to end-stage disease. Another four patients died of right ventricular infarction. Causes of death in the

TABLE 3 Autoimmune serology according to the diffusing capacity of the lung for carbon monoxide (DLCo)

\begin{tabular}{|c|c|c|c|}
\hline Autoimmune serology & DLco $<45 \%$ pred $^{\#}$ & $D\llcorner C O \geqslant 45 \%$ pred & p-value \\
\hline Antinuclear antibodies & $13(38)$ & $16(20)$ & 0.063 \\
\hline Anti-double stranded DNA & $0(0)$ & $1(2)$ & 0.998 \\
\hline Antineutrophil cytoplasmic antibody & $0(0)$ & $6(10)$ & 0.998 \\
\hline
\end{tabular}

Data are presented as $n(\%)$. The $p$-values are from binary logistic regression analysis corrected for age. ${ }^{\#}: n=34 ;{ }^{\top}: n=81$. 
TABLE 4 Clinical characteristics associated with a severely reduced diffusing capacity of the lung for carbon monoxide on multivariate regression analysis

\begin{tabular}{lccc} 
Clinical characteristic & OR & $\mathbf{9 5 \%} \mathbf{C l}$ & p-value \\
\hline Age years & 1.73 & $1.27-2.37$ & 0.001 \\
Pack years & 1.35 & $1.07-1.71$ & 0.016 \\
\hline
\end{tabular}

$\mathrm{OR}$ and $95 \% \mathrm{Cl}$ are presented per 10 years.

remaining three patients were multi-organ failure, left ventricular infarction and intrapulmonary bleeding. Event-free survival was lower in $\mathrm{IPAH}_{<45 \%}$ when compared to IPAH $\mathrm{IP}_{\geqslant 45}$ (log-rank $\left.\mathrm{p}<0.001\right)$. A severely reduced DLCO was associated with event-free survival univariately (hazard ratio (HR) 3.80, 95\% CI 2.20-6.58; $\mathrm{p}<0.001$ ), but not when controlled for age at diagnosis, sex, time between the DLCO measurement and diagnosis and the presence of coronary disease (HR 1.78, 95\% CI 0.91-3.50; $\mathrm{p}=0.09$ ).

\section{Discussion}

Here, we not only confirm that the presence of a severely reduced DLCO in IPAH is associated with a poor survival $[8,9]$, but also that in a large cohort of I/HPAH patients a low DLCO is associated with a higher age and a higher tobacco exposure, more CT abnormalities and a worse exercise performance; despite similar haemodynamic profiles. We confirm the decreased survival in this patient group and now show that this poor outcome is related to age, sex and the presence of coronary disease.

The lack of correlation between DLCO and haemodynamic parameters, such as PVR and CO, confirms findings by others $[1,17]$. Hence, the results of this study do not suggest that a severe reduction in DLCO is the result of IPAH alone, but rather that a severe reduction in DLCO identifies a subtype of IPAH patients in whom the disease is possibly related to smoking.

\section{Parenchymal lung disease}

Cigarette smoking is known to be a risk factor for the development of emphysema and interstitial lung disease (ILD) $[18,19]$. Both emphysema and ILD are known to reduce the DLCO and the presence of pulmonary hypertension $(\mathrm{PH})$ further enhances this reduction $[6,15]$. In our study, severe emphysema and/or evident ILD were excluded at the time of diagnosis as well as after re-evaluation of the HRCT, which had been routinely performed during the diagnostic work-up. Consistent with previous studies, a substantial number of IPAH patients had mild-to-moderate degrees of emphysema or interstitial abnormalities on HRCT $[4,20]$. As the severity of emphysema on HRCT correlates inversely with DLCO [21], mild-to-moderate emphysema will probably only moderately reduce the DLCO. Likewise, mild parenchymal abnormalities are known to only slightly reduce DLCO [22, 23]. Smoking appears to augment this reduction in DLCO [23]. It is possible that in some IPAH patients, additive effects of smoking, mild or moderate interstitial lung abnormalities and/or emphysema together explain a severe reduction in DLCO. This is supported by the higher prevalence of these abnormalities observed in IPAH patients with a severely reduced DLCO when compared to IPAH patients with a more preserved DLCO.

The CT findings also indicate that in our study cohort the presence of mild forms of the syndrome of combined pulmonary fibrosis and emphysema (CFPE) could not be excluded. The typical features of CFPE, such as older age, male sex, smoking history and a relatively preserved pulmonary function in the presence of a severely reduced DLCO [7], were also present in our IPAH patients with a severely reduced DLCO. It is currently unclear whether patients with severely increased PAP) and mild-to-moderate parenchymal abnormalities with preserved pulmonary function should be diagnosed and treated as IPAH patients or as patients with lung disease associated (but "out of proportion") $\mathrm{PH}$.

\section{Smoking-related pulmonary vasculopathy}

Tobacco exposure was independently associated with a severe reduction in DLCO. Therefore, IPAH patients with a severely reduced DLCO may represent a subgroup of PAH patients with a smoking-related distinct pulmonary vasculopathy $[24,25]$.

Previous studies have shown that tobacco exposure alone can cause pulmonary vascular remodelling leading to $\mathrm{PH}[24,25]$. An experimental animal study also showed that the degree of PAP elevation caused by smoking is similar to that provoked by hypoxia, but that the underlying mechanism differs between the two types of exposure [24]. It can be hypothesised that smoking, in IPAH patients with a severely reduced DLCO, 

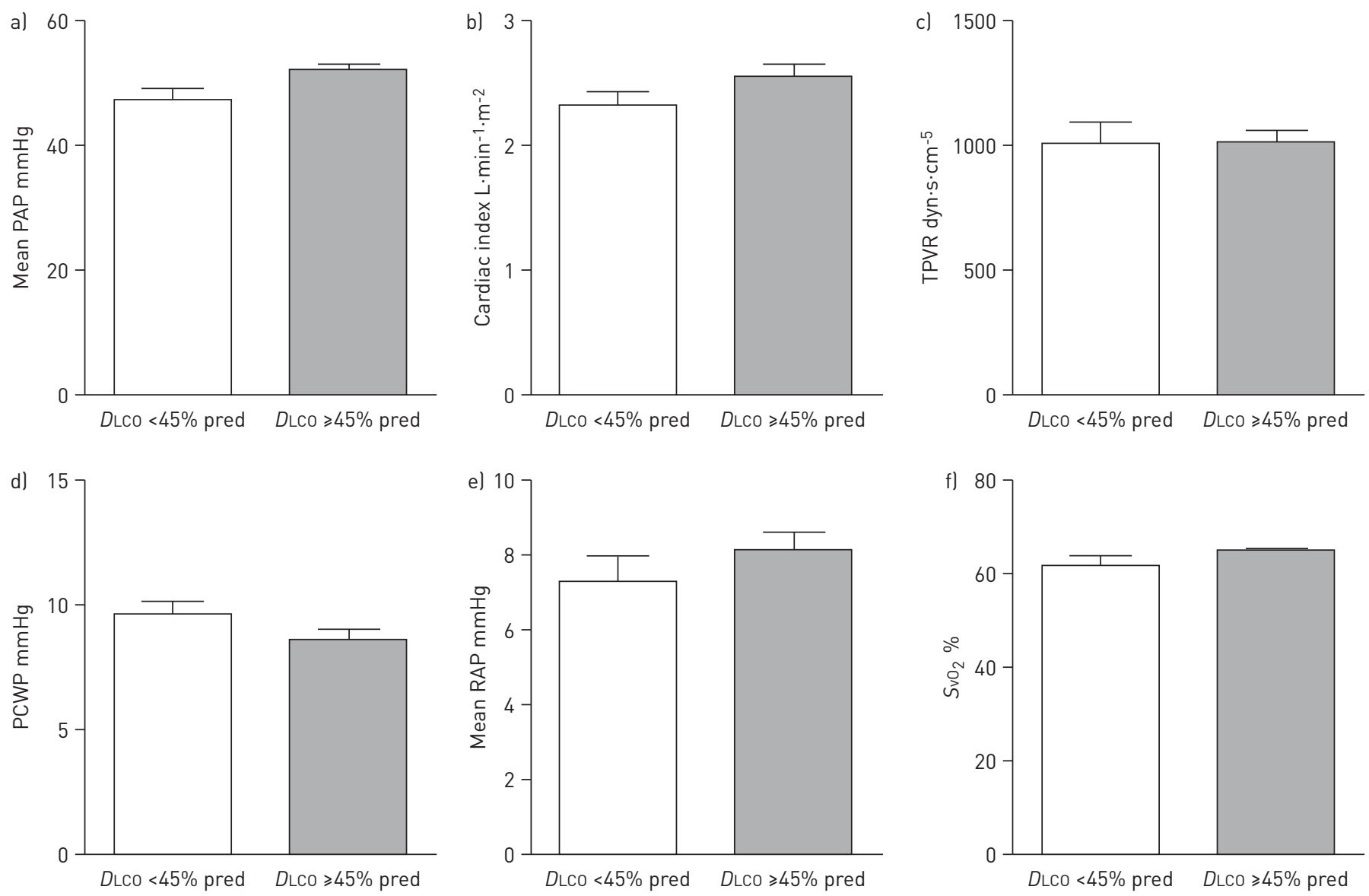

FIGURE 2 Haemodynamic parameters at the time of diagnosis of pulmonary arterial hypertension (PAH) according to diffusion capacity. a) Idiopathic PAH patients with a severely reduced diffusing capacity of the lung for carbon monoxide (DLCO) had similar mean pulmonary arterial pressure (PAP) when corrected for age. No differences were observed in b) cardiac index, c) total pulmonary vascular resistance (TPVR), d) pulmonary capillary wedge pressure (PCWP), e) mean right atrial pressure (RAP), or $\mathrm{f})$ mixed venous oxygen saturation $\left(\mathrm{SvO}_{2}\right)$. Data are presented as mean \pm SEM.

vascular lesions caused by smoking are predominantly present. However, it is unclear whether these vascular lesions could fully explain a severe reduction in DLCO or whether additional parenchymal damage or interstitial thickening is required.

Interestingly, patients with a severe reduction in DLCO were relatively more frequently males and male sex may have interacted with the effects of smoking on the lung parenchyma or vasculature [26-28], together inducing a severe reduction in DLCO. Male smokers may be more susceptible than female smokers to develop PAH, as suggested by a case-control study that was conducted in Switzerland. In that study, the prevalence of smoking was higher in male PAH patients than in the general population, while in females the prevalence of smoking was similar [26].

\section{Pulmonary veno-occlusive disease}

The IPAH patients with a severely reduced DLCO in this study share some similarities with patients with PVOD. First, DLCO is severely reduced as is the case in PVOD [29]. Secondly, a lower $\mathrm{SaO}_{2}$, a similar sex distribution, increase in tobacco exposure and 6MWD is found in our subgroup of IPAH patients [29]. The amount of similarities between the two groups suggests that some IPAH patients with a severe DLCO reduction may actually have PVOD. This is supported by histopathological review studies on IPAH patients showing that $\sim 10 \%$ of patients with a clinical diagnosis of IPAH were found to have PVOD [13].

However, we consider it unlikely that all IPAH patients with a severely reduced DLCO are actually PVOD patients. We excluded all patients who were suspected of PVOD based on either clinical criteria $[4,20]$ or histopathological evidence. This gives a PVOD prevalence of $7 \%$ in our IPAH population, which is comparable to the estimated prevalence of $10 \%$ [13]. All of these patients were excluded from further analysis. Nevertheless, we cannot exclude that some PVOD patients present with a different, non-classical profile and are, therefore, wrongly diagnosed as IPAH. Although non-classical PVOD may explain a low 

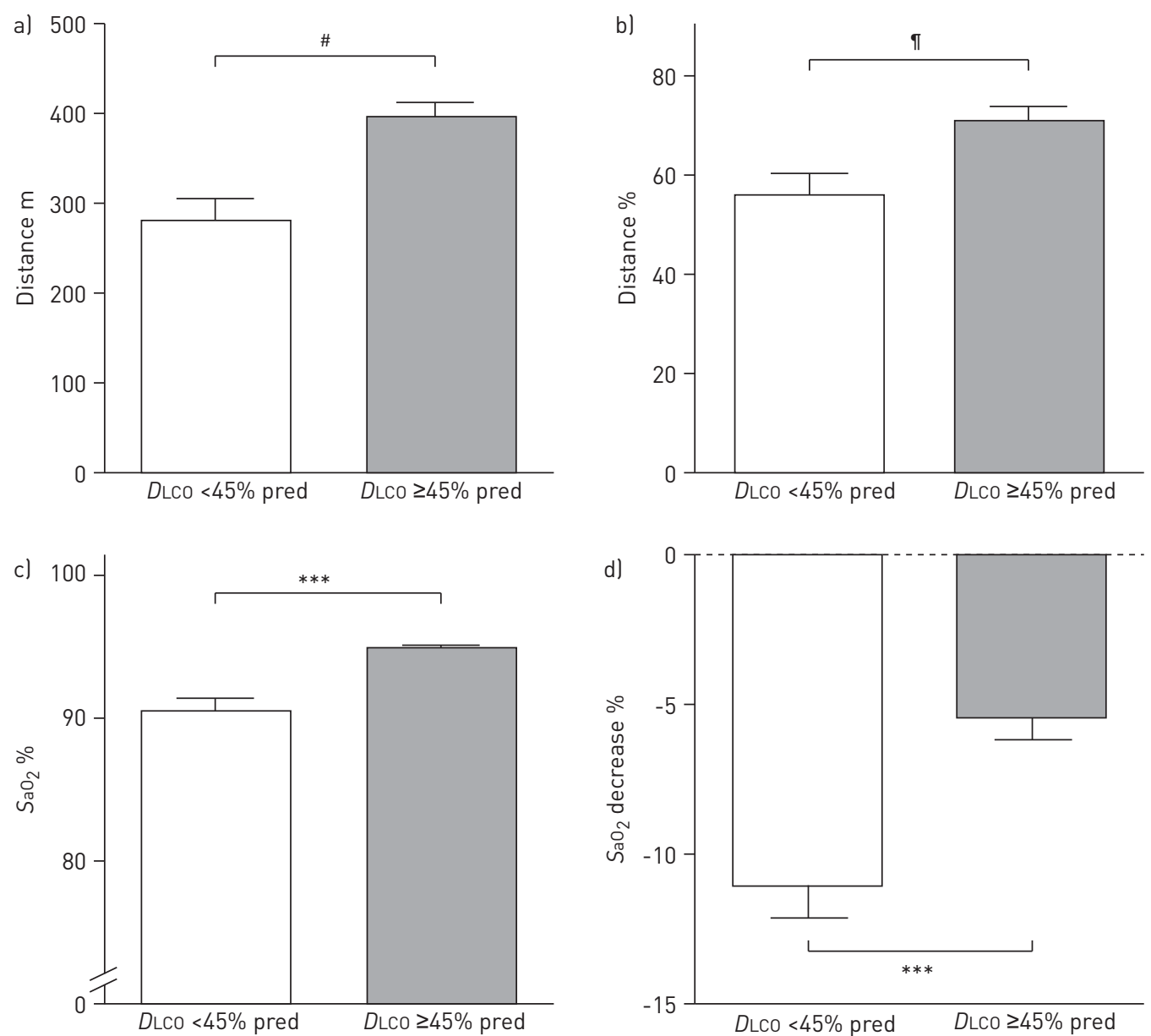

FIGURE 3 6-min walk test results at baseline according to the diffusing capacity of the lung for carbon monoxide (DLCO) groups. Idiopathic pulmonary arterial hypertension (IPAH) patients with a severely reduced DLCO walked less far, as shown by distance in metres and by distance as percentage of predicted $(a, b)$. IPAH patients with a severely reduced DLCO had lower arterial oxygen saturation $\left(\mathrm{SaO}_{2}\right)$ at rest $(\mathrm{c})$ and a larger decrease in saturation during exercise (d). Data are presented as mean \pm sEM. ${ }^{*}: \mathrm{p}=0.034 ;{ }^{\uparrow}: \mathrm{p}=0.003 ;{ }^{* *}: \mathrm{p}<0.001$.

DLCO in some patients, it is unlikely that this serves as an explanation for the entire group of low DLCO patients. Further histopathological studies are required in this specific cohort to study the nature of the pulmonary vascular lesions.

\section{Left heart disease}

A severely reduced DLCO is found in $\sim 25 \%$ of patients with PH due to left heart failure with preserved ejection fraction [5]. Therefore, a possible explanation for a severe reduction in DLCO in IPAH may be the presence of left heart failure. However, overt left heart failure is very unlikely, as in our study cohort no patients were included with increased PCWP or evident left ventricular dysfunction on echocardiography. In addition, no differences in PCWP could be observed between the two groups, strongly indicating that there were no differences in left heart function at rest. We did not routinely perform fluid challenge or exercise during right heart catheterisation as these interventions are not part of the current diagnostic algorithm. Consequently, we cannot exclude the existence of occult diastolic dysfunction. We observed an increased prevalence of coronary artery disease in IPAH patients with a low DLCO. As this finding was not independently predictive of a low DLCO, this finding may rather reflect the increased tobacco exposure of IPAH patients with a low DLCO. Future studies on PCWP changes after fluid challenge or exercise could identify patients with diastolic dysfunction. Whether these patients require a different treatment strategy remains to be established.

\section{Clinical implications}

IPAH is usually considered a homogenous disease, but here we show that within the IPAH population heterogeneity can be observed. We characterised a subgroup of IPAH patients with a severely reduced DLCO 
TABLE 5 First-line treatment, treatment response and survival

\begin{tabular}{|c|c|c|c|}
\hline & DLCO $<45 \%$ pred & $D$ LCO $\geqslant 45 \%$ pred & p-value \\
\hline First-line treatment & & & 0.238 \\
\hline None & $0(0)$ & $2(2)$ & \\
\hline $\mathrm{Ca}^{2+}$ channel blockers & $3(6)$ & $13(11)$ & \\
\hline Endothelin receptor antagonist & $23(48)$ & $42(36)$ & \\
\hline Prostanoids & $9(19)$ & $32(27)$ & \\
\hline Phosphodiesterase inhibitors & $11(23)$ & $15(13)$ & \\
\hline Combination therapy & $2(4)$ & $13(11)$ & 0.235 \\
\hline \multicolumn{4}{|l|}{ Treatment response } \\
\hline Time to clinical worsening days & $435(153-868)$ & $592(315-1522)$ & $0.539^{\circ}$ \\
\hline Survival years & & & $0.002^{\circ}$ \\
\hline 1 & 87 & 95 & \\
\hline 3 & 54 & 86 & \\
\hline 5 & 38 & 80 & \\
\hline
\end{tabular}

Data are presented as $\mathrm{n}(\%)$, median (25th-75th percentile) or \%, unless otherwise stated. * : time to clinical worsening is defined as time to add-on therapy or an event latrial balloon septostomy, lung transplantation or death); ${ }^{\top}$ : p-value corrected for age.

and showed that this group has a distinct clinical profile with presentation at higher age, a relative over representation of male patients, a greater tobacco exposure, an increased prevalence of coronary disease and, more often, abnormalities on chest CT scan. Remarkably, not one patient with a severely reduced DLCO had heritable disease. These findings suggest that the group of IPAH patients with a severely reduced DLCO is a mixed population and may contain patients with occult diastolic dysfunction, patients with parenchymal lung disease not severe enough to be classified in the WHO FC III of the Dana Point classification [12], and perhaps patients with non-classical PVOD. Our subgroup of IPAH patients shares similarities with the older IPAH patient population of the COMPERA registry (Prospective Registry of Newly Initiated Therapies for Pulmonary Hypertension) [11]. This registry had a predominance of elderly patients showing that the demographics of IPAH patients are changing. With the IPAH population getting older, heterogeneity will probably increase and our subgroup of IPAH patients with a severely reduced DLCO will probably be of great relevance in the future. Our findings raise the question whether within the IPAH population as a whole, subgroups exist that may require a different diagnostic and/or therapeutic strategy.

\section{Study limitations}

Our study was retrospective in nature and some data were missing in individual patients. The number of missing values did not exceed $10 \%$ in the majority of parameters studied. However, in three parameters that were considered to be possible contributors to a severely reduced DLCO, we found the number of missing values to exceed the 10\%: which included: 1) the amount of pack years; 2) cardiac output; and 3) pulmonary vascular resistance. Therefore, the lack of an association of CO or PVR with DLCO cannot be ruled out based on the present study. Because $>90 \%$ of data was available for the majority of parameters studied, we consider it to be unlikely that the nature of the study explains the finding of a distinct clinical profile in the low DLCO group.

Pulmonary function test results used to divide the IPAH patients into two DLCO groups were not all measured at baseline. However, we observed that DLCO did not change over time. Indeed, only two patients with a severely reduced DLCO, and four patients with a more preserved DLCO changed DLCO, which meant that they also changed DLCO group. Therefore, the time of DLCO measurement presumably did not influence our results.

We used TTCW to determine treatment response. With our broad inclusion period the definition of TTCW may not be a good reflection of true worsening due to the differences in available PAH specific drugs between early and recent diagnosed patients.

We included both IPAH and HPAH patients diagnosed between 1990 and 2011. This is a broad inclusion period and several changes in IPAH patient's characteristics have occurred over the last decades [10]. Therefore, it could be that the IPAH patient groups do not completely represent the incident cases of IPAH and hereditary PAH seen today. However, as our subgroup of IPAH patients, with a severely reduced DLCO, share many characteristics with the newly diagnosed older IPAH patients described by both LING et al. [10] 
and Hoeper et al. [11], we believe our subgroup is still of relevance today and may even be of greater relevance in the future.

\section{Conclusions}

We show that a severe reduction in diffusion capacity in IPAH is associated with a higher age at presentation, a greater tobacco exposure and a poor exercise performance, despite a haemodynamic profile that is not different from other IPAH patients. We confirm the decreased survival in this patient group and now show that this poor outcome is related to age, sex and the presence of coronary disease.

\section{References}

1 Sun XG, Hansen JE, Oudiz RJ, et al. Pulmonary function in primary pulmonary hypertension. J Am Coll Cardiol 2003; 41: 1028-1035.

2 Kawut SM, Taichman DB, Archer-Chicko CL, et al. Hemodynamics and survival in patients with pulmonary arterial hypertension related to systemic sclerosis. Chest 2003; 123: 344-350.

3 Overbeek MJ, Groepenhoff H, Voskuyl AE, et al. Membrane diffusion- and capillary blood volume measurements are not useful as screening tools for pulmonary arterial hypertension in systemic sclerosis: a case control study. Respir Res 2008; 9: 68.

4 Montani D, Achouh L, Dorfmüller P, et al. Pulmonary veno-occlusive disease: clinical, functional, radiologic, and hemodynamic characteristics and outcome of 24 cases confirmed by histology. Medicine 2008; 87: 220-233.

5 Agarwal R, Shah SJ, Foreman AJ, et al. Risk assessment in pulmonary hypertension associated with heart failure and preserved ejection fraction. J Heart Lung Transplant 2012; 31: 467-477.

6 Behr J, Ryu JH. Pulmonary hypertension in interstitial lung disease. Eur Respir J 2008; 31: 1357-1367.

7 Cottin V, Le Pavec J, Prévot G, et al. Pulmonary hypertension in patients with combined pulmonary fibrosis and emphysema syndrome. Eur Respir J 2010; 35: 105-111.

8 Benza RL, Miller DP, Gomberg-Maitland M, et al. Predicting survival in pulmonary arterial hypertension: insights from the Registry to Evaluate Early and Long-Term Pulmonary Arterial Hypertension Disease Management (REVEAL). Circulation 2010; 122: 164-172.

9 Chandra S, Shah SJ, Thenappan T, et al. Carbon monoxide diffusing capacity and mortality in pulmonary arterial hypertension. J Heart Lung Transplant 2010; 29: 181-187.

10 Ling Y, Johnson MK, Kiely DG, et al. Changing demographics, epidemiology and survival of incident pulmonary arterial hypertension. Am J Respir Crit Care Med 2012; 186: 790-796.

11 Hoeper MM, Huscher D, Ghofrani HA, et al. Elderly patients diagnosed with idiopathic pulmonary arterial hypertension: results from the COMPERA registry. Int J Cardiol 2013; 168: 871-880.

12 Galiè N, Hoeper MM, Humbert M, et al. Guidelines for the diagnosis and treatment of pulmonary hypertension. Eur Respir J 2009; 34: 1219-1263.

13 Macintyre N, Crapo RO, Viegi G, et al. Standardisation of the single-breath determination of carbon monoxide uptake in the lung. Eur Respir J 2005; 26: 720-735.

14 Quanjer PH, Tammeling GJ, Cotes JE, et al. Lung volumes and forced ventilatory flows. Report Working Party Standardization of Lung Function Tests, European Community for Steel and Coal. Official Statement of the European Respiratory Society. Eur Respir J 1993; 6: Suppl. 16, 5-40.

15 Jing ZC, Xu XQ, Badesch DB, et al. Pulmonary function testing in patients with pulmonary arterial hypertension. Respir Med 2009; 103: 1136-1142.

16 Balbi B, Cottin V, Singh S, et al. Smoking-related lung diseases: a clinical perspective. Eur Respir J 2010; 35: 231-233.

17 Lederer DJ, Enright PL, Kawut SM, et al. Cigarette smoking is associated with subclinical parenchymal lung disease: the Multi-Ethnic Study of Atherosclerosis (MESA)-lung study. Am J Respir Crit Care Med 2009; 180: 407-414.

18 Resten A, Maitre S, Humbert M, et al. Pulmonary hypertension: CT of the chest in pulmonary venoocclusive disease. AJR Am J Roentgenol 2004; 183: 65-70.

19 Klein IS, Gamsu G, Webb WR, et al. High-resolution CT diagnosis of emphysema in symptomatic patients with normal chest radiographs and isolated low diffusing capacity. Radiology 1992; 182: 817-821.

20 Gochuico BR, Avila NA, Chow CK, et al. Progressive preclinical interstitial lung disease in rheumatoid arthritis. Arch Intern Med 2008; 168: 159-166.

21 Rosas IO, Ren P, Avila NA, et al. Early interstitial lung disease in familial pulmonary fibrosis. Am J Respir Crit Care Med 2007; 176: 698-705.

22 Ferrer E, Peinado VI, Castaneda J, et al. Effects of cigarette smoke and hypoxia on pulmonary circulation in the guinea pig. Eur Respir J 2011; 38: 617-627.

23 Santos S, Peinado VI, Ramirez J, et al. Characterization of pulmonary vascular remodelling in smokers and patients with mild COPD. Eur Respir J 2002; 19: 632-638.

24 Schiess R, Senn O, Fischler M, et al. Chest 2010; 138: 1086-1092.

25 Sverzellati N, Calabrò E, Randi G, et al. Sex differences in emphysema phenotype in smokers without airflow obstruction. Eur Respir J 2009; 33: 1320-1328.

26 Washko GR, Hunninghake GM, Fernandez IE, et al. Lung volumes and emphysema in smokers with interstitial lung abnormalities. N Engl J Med 2011; 364: 897-906.

27 Montani D, Kemp K, Dorfmuller P, et al. Idiopathic pulmonary arterial hypertension and pulmonary venoocclusive disease: similarities and differences. Semin Respir Crit Care Med 2009; 30: 411-420.

28 Mandel J, Mark EJ, Hales CA. Pulmonary veno-occlusive disease. Am J Respir Crit Care Med 2000; 162: 1964-1973.

29 Montani D, Price LC, Dorfmuller P, et al. Pulmonary veno-occlusive disease. Eur Respir J 2009; 33: 189-200. 